九州大学学術情報リポジトリ

Kyushu University Institutional Repository

\title{
Semiotics of Void and Information Representation
}

Tanaka-Ishi i, Kumiko

Dept of Advanced Info Tech, ISEE, Kyushu University : Professor :

http://hdl. handle. net/2324/27215

出版情報: Proceedings of the 15th International Conference on Human-Computer Interaction (HCI). 8012, pp. 152-161, 2013. Springer

バージョン:

権利関係: (C) Springer-Verlag Berlin Heidelberg 2013 


\title{
Semiotics of Void and Information Representation
}

\author{
Kumiko Tanaka-Ishii \\ Kyushu University \\ kumiko@ait.kyushu-u.ac.jp
}

\begin{abstract}
The objective of this article is to present a semiotic analysis of voidwhich in this article is the spacio-temporal empty space existing in any representation - in order to consider the representation of quality and to show how this is essential in human representation yet difficult to process computationally. First, a summary of reference to void is presented through a comparison between Western and Eastern cultural approaches to void. A semiotic model of void is then developed by applying both Saussurian and Peircian frameworks and explaining how the two frameworks become equivalent when applied to void, as well as how void is essentially a structural entity. After analysis of various semiotic kinds of void, the article examines the difficulty of computational handling of void and suggests possible paths towards a more human-oriented form of information representation.
\end{abstract}

Keywords semiotics, information representation, void, structure, index, icon, design

\section{Void in Information Representation}

Void in this article signifies the spacio-temporal empty space existing in a representation. Spatially, void indicates the empty spaces among content. For example, every character space in this article, the space framing the text, and the space between lines are all part of the void. Temporally, void consists of the non-event periods between consecutive temporal events.

From this definition, void exists everywhere, in any kind of spacio-temporal representation. The design of void partly defines how well the representation as a whole communicates. It requires consideration of the size, frequency, and density of void among non-void occurrences. Such representations naturally include any computational representation, inclusively of all automatic syntheses. For example, the reason why people find most automatic syntheses mechanical partly lies in the lack of naturalness in the design of void. 


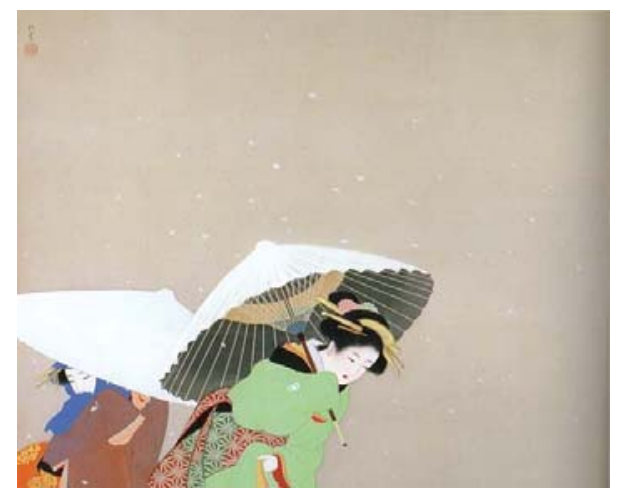

Figure 1: Uemura Shōen, Feathered Snow, Color on Silk, Shōwa Period, 1944 Yamatane Museum of Art, Tokyo, Japan. Original in color.

Void at first seems no more than something left out after articulating content or an event, and thus unworthy for analysis. Signification by void, such as the naturalness noted above, however, suggests that void itself speaks. Consideration of a representation that communicates well cannot leave void literally as emptiness without any concrete content. In other words, void in a representation has meaning or at least adds meaning to non-void content and events.

The first objective of this article is to consider void semiotically. Semiotics is adopted here as the theoretical basis for analysis of any media with meaning. Such semiotic analysis of void would provide insights on the essential differences between human and computational semiotic systems. This understanding would naturally push us to seek better information representation and design. This leads to the question, however, of better in which way? Hence, this article examines this question with respect to HCI and UX after brief reference to the previous conjecture on void and semiotic modeling of void.

\section{West vs. East: A Brief Summary of Previous References to Void}

The historical focus on the notion of void contrasts between the West and East. The West, with the theoretical traditions of ancient Greek philosophy and Christianity, valued rational minds which typically required classifying an analysis target into a few components. Since clarity was valued, void, which tends to remain ambiguous, was avoided. This led to the tendency of void not to develop as a subject itself, with the result that either nothing is left blank or blankness is considered something leftover and unworthy for analysis. For example, Western paintings before the modern era have the strong tendency to fill the whole canvas, as established through the study of perspective, which is the effect obtained by a representation exterior to the subject estate. 
In contrast, in the East the notion of void has been considered central in different kinds of representation. This is supported by the philosophical background of Buddhism, in which void is considered the source of holism from which everything is generated. Ambiguity has not been considered something to be avoided. In Eastern representation, the question of how to organize blankness often became a subject itself.

In Japan, void is described through the term '間' (read ma, literally meaning something in between), and the notion has been considered important through every kind of representation. Figure 1 shows one example of painting making use of void. Minami in [6] indicates how the notion of ma governs every Japanese cultural representation, including language, painting, poetry, theater, film, ritual, music, dance, architecture, and martial arts. Moreover, the notion of $m a$ appears in various Japanese words. For example, the term mistake in Japanese is ma-chigai whose literal translation is $m a$ taken incorrectly. In China, as well, the mention of void has a long history with respect to Lao-zi's philosophy of Taoism [2]. The notion developed mainly through the term '无' (read Wú, meaning none), although there are other related terms such as '空' (read kong, meaning empty), as raised by [2].

The two contrastive cultural approaches of East and West towards void, however, have became closer under the influence of globalization. From the Western side, one typical example can be seen in a modern trend in cognitive science, through the distinction of figure and ground. Originally, the ground remained the less interesting estate, but Gestalt psychology then indicated how the figure and ground can get reversed [4]. In parallel to such increased focus on targets which had acquired less focus in the West, the East has also acquired a great influence of Western rationalism, resulting in less emphasis on traditional Eastern cultural notions. Within this current, the mention of void seems to have decreased, resulting in rare consideration within frameworks of an international, academic, theoretical basis, including semiotics. Even when void is mentioned, the argument entails analysis of a specific Eastern artistic genre, as seen for Chinese painting [2], garden rock placement [1], or art related to Buddhism [3].

Before going on to semiotic consideration of void, note that there is a question of whether voids with different cultural backgrounds can be considered as one. For example, voids in China and Japan are not identical, as seen from how they are primarily articulated. Briefly, Chinese void has a more absolute notion, considered as the source of the yin-yang contrast in Chinese philosophical thought, whereas Japanese void is more relative to other, non-void existences. Even with such different cultural backgrounds, both types of voids influenced each other throughout history. Precisely speaking, every void has a cultural color, and analysis of the different notions would be interesting. In this article, however, in addition to the limitation of space, since the context lies in information representation, which currently concerns every culture, I consider the universality of void in an abstract manner. 


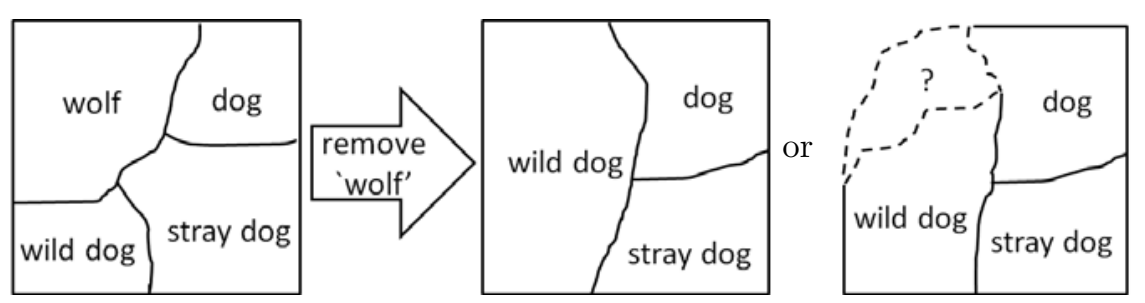

Figure 2: Saussurian traditional semiotic field formed from relations among signs, filling the semantic field taken from [5] (left, center) and an alternative (right)

\section{Semiotic Modeling of Void}

Modeling void by the theory of semiotics in fact is challenging, since the focus of semiotics is on what is articulated in the form of a sign. Void at first only seems something left out, the remaining resource after some articulation is made. Thus, considering void as a sign could even sound contradictory. Partly because of such contradiction, void has rarely been considered to form a semiotic target, even though a substantial variety of representation targets have been considered within semiotics [7]. Some exceptions exist, as found in [2], which suggests considering void (le vide in Cheng, i.e., emptiness) by means of semiology, but even here the application of fundamental semiotic theories to the notion of void has been limited, made only through analysis of Chinese paintings.

\subsection{Model of Void}

First of all, we must consider the plausibility of considering void as a kind of sign. Above all, Saussure emphasized how the meanings of signs are defined relatively within the holistic system of value such that each sign cannot exist absolutely. Saussure raises an example of wolf and dog to explain this in [9]. Maruyama illustrates this idea as shown in Figure 2 (left), in which every area surrounded by a contour is considered as an articulated sign [5][page 96]. If one of the signs (e.g., wolf) did not exist or was removed, the signs placed next to the original would take its place (center), by changing the contour of the semantics of the other signs, such as dog and wild dog.

This image well illustrates the relativity of the values of signs. When one sign is removed, however, should the whole semantic field be completely filled? If the notion of "wolf" did not exist or disappeared, wouldn't there be the possibility of having a semantic portion in which wolves are considered as unknown animals, different from both wild dogs and dogs? Moreover, how could this relative change in the notion be explained in relation to the social convention having the effect of stabilizing the semantic meaning of a sign? I consider that the Saussurian notion of relativity does not necessarily mean that the semantic field should be completely filled. Rather, it could leave space for something 

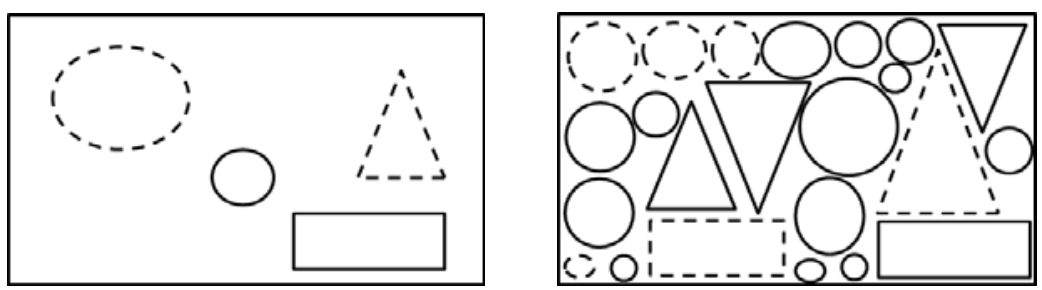

Figure 3: Semiotic fields with voids

unknown or undefined. This can be represented by changing both the contours of every sign and the contour of the whole semantic field, with some semantic borders left obscure, as denoted by the dashed lines in the rightmost figure.

Upon allowing such "left out" or unknown spaces in the semantic field, we find out that these spaces do possess meaning by themselves, as illustrated in Figure 3. Here, objects with various shapes represent non-void signs, and the left-out spaces represent undefined spaces existing within the field. The left-out spaces could have obscure borders (denoted by dashed lines), as seen previously. Comparing the two figures in Figure 3, anyone would feel that in the right figure, the objects (i.e., the non-void signs) are more densely placed, suggesting a further connotation that the semiotic space is more tightly packed than that on the left. This could suggest further, for example, how the semantic domain either has much left to be studied (left) or is well established (right), depending on the target field. Even from this simple example, we see that void generates meaning. The void in Figure 1 also provokes imagination of the cold, dim, cloudy winter sky, which effectively highlights the ladies' clothing colors.

Void thus can be considered to form a sign and become a target of semiotics. Indeed, in the East, the notion of void has been considered through a sign, 間 or 无. Even without verification, [2] naturally considered le vide within the context of semiotics. In the Saussurian sign model, the signifier is deemed the representation of void, and the meaning raised through the existence of void constitutes the signified. For example, in the case of Figure 1, the signifier is deemed the blank space, whereas the meaning raised through imagination (the dim sky, the effect of highlighting the clothing, etc.) forms the signified.

Comparing void and non-void signs, void as a sign has two characteristics. First, it influences the context, or the system, directly. In the same example of Figure 1, the void part is defined by the placement of other non-void signs (namely, the ladies). The final setting of void constitutes the relation or the context of the other non-void signs. Such directness in relation to the sign system as a whole characterizes void. Saussure emphasized how the meaning of a sign should be considered within the holistic system of the sign. Void, if admitted to consideration as a sign, is then a typical sign that directly concerns the whole semiotic field, the holistic semiotic system itself.

Second, void is defined through its use. Void is the space that remains after placing other non-void signs within void itself. This explanation itself shows how 


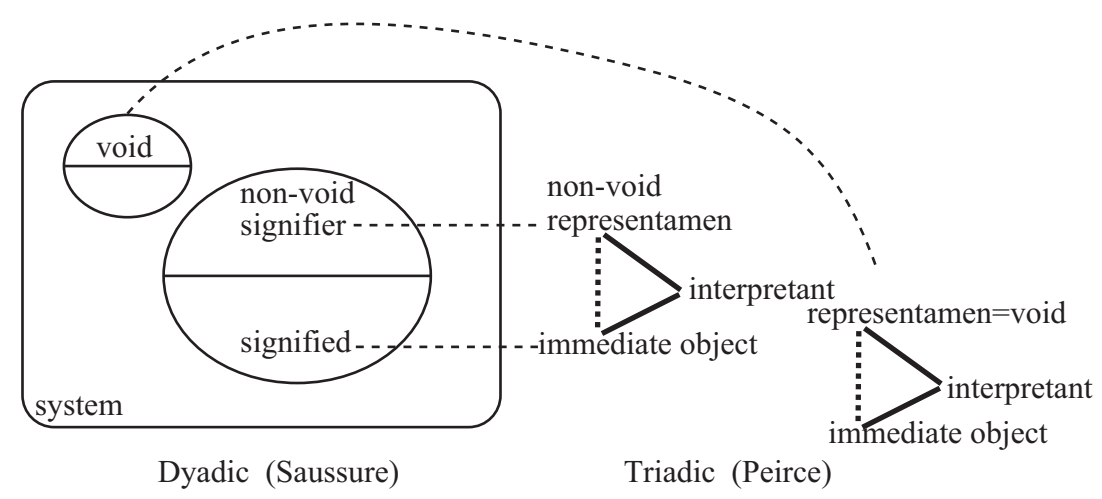

Figure 4: Void as a sign

void is defined self-referentially. In the same example of Figure 1, the placement of other non-void signs is the use of blank area, which defines what and how void exists here. The painter must have searched for the optimal composition in terms of use of void, and the final choice defines the content of the void. The reason why void directly influences the system lies in its way of being defined through its use. In other words, void is a self-referential sign, which acquires its content through the use of itself.

So far, we have considered Saussure's framework. Alternatively, Peirce's semiotic framework can model such reflexivity in semiotics. In [11], I argued how the Peircian and Saussurian frameworks might correspond differently from what had been believed, through analysis of different computer programming paradigms. According to this conclusion, by considering Peirce's dynamical object to correspond to Saussure's thing, two relata of the Peircian triadic sign, the representamen and immediate object, correspond to the relata of the Saussurian dyadic sign, the signifier and signified. The third of Peirce's relata, the interpretant, is found in the Saussurian framework in the form of difference.

This new correspondence applies naturally to a semiotic discussion of void as a sign, as shown in Figure 4. The left side of the figure shows the Saussurian model, in which there are two signs, void and non-void. In a usual semiotic representation, there could be multiple non-void signs related to void and other non-void signs. Void as a sign forms the context of the non-void signs, and therefore, void relates to non-void, within the semiotic system; in other words, void forms difference. In applying the Peircian triadic framework, on the other hand, as shown on the right side of the figure, void would form part of the interpretant, as the use of the non-void signs. The dashed lines indicate the correspondences of the relata in each framework.

In [11], I further indicated how a sign whose content (i.e., the signified, or Peirce's immediate object) is defined through its use (i.e., within the Saussurian system, or through Peirce's interpretant) makes the two different models equivalent. A self-referential sign acquires its content through the use of itself; 
therefore, content and use become tightly coupled, because articulation requires reference to a complex unit operating as the self through a signifier. Void is yet another example of a self-referential sign to which such equivalence applies.

This reciprocal nature of void further suggests that it is something human and difficult to handle naturally within a computational information representation. Before going on to this aspect, however, it is interesting to see what kinds of void exist, through semiotic consideration of different kinds of sign.

\subsection{Kinds of Void}

If void can be considered as a sign, it should be classifiable in terms of semiotic classes. Such a conjecture could provide a better understanding of the nature of void. In the frameworks of both Saussure and Peirce, such sign classes have been proposed [7], for the former by Hjelmslev and the latter by Peirce himself. I discussed their correspondence in [11], at least through signs used in programming languages. Therefore, either framework should apply, given these discussions, and I have chosen to apply the most widely used sign classes framed by Peirce [8] (i.e., icon, index, symbol) to consider the nature of void. Peirce's definitions of the icon, index, and symbol are as follows [8]:

- An icon is "a sign which stands for something merely because it resembles it" [3.362], "partaking in the characters of the object" [4.531]. For example, a portrait of a person or a color sample of paint is an icon since it stands for something merely because it resembles the original.

- An index is "physically connected with its object; they make an organic pair, but the interpreting mind has nothing to do with this connection" [2.299]; "it is a "reference" " [2.283]. According to Peirce, clocks, sundials, and door knocks are example of indexes, since each makes an organic pair with another fact. Moreover, uses of signs A, B, and C in a formal statement such as 'A and B are married and C is their child' are indexes [2.285].

- A symbol is a sign that "refers to the object that it denotes by virtue of law, usually an association of general ideas" [2.249] "Any ordinary word as 'give', 'bird', 'marriage', is an example of a symbol" [2.298].

Briefly, in the jargon of computer science, an icon represents an instance, an index represents a variable, and a symbol represents a type.

Void as icon corresponds to the specific blanks and spaces introduced for a representation, as instances of spacing. For example, a visual representation requires regulation of space before it is complete, and this could require modification applied to the general spacing rules governing the representation. Such adjustment is often due to optical illusions caused by the placement of non-void content. Another example could be a moment of silence in a musical performance. The best length depends on the context, as the performance, its speed and intensity, and every musical non-event moment constitutes an instance.

Void as index requires attachment of a secondary, unrelated meaning, according to Peirce's definition. An example was already shown in Figure 1, in which the space is not only a blank but also requires the viewer's imagination. 
Another temporal example is Cage's piece 4'33". The "performance" of silence in this piece provokes consideration of what is music, and such connotation is indexed through the void as silence. From this view, an artistically designed representation of void is deemed an index, with the difference from icon being whether the void adds a further connotation.

Lastly, void as Peircian symbol is deemed to indicate the typical types of spaces in a representation. We often refer to such voids in terms of format. A text format (such as the format for this paper, specified by the HCII Conference) defines spacing, or how void should appear together with text: the amount of space between lines, and the blank spaces framing the main text. The normal lengths of musical notes indicated in musical scores also define such a format, although the notes become icons once played (if played by a human).

Such discussion of void also supports the notion of how void could constitute a sign and be analyzed within a semiotic framework. Moreover, this discussion also shows another characteristic of having interest in void as index or icon, rather than as a Peircian symbol. Usually in semiotics as a science, the emphasis of such classification lies more in organization in the form of symbols, i.e., a typical group of signs appearing in a certain kind of representation. In contrast, the question of void governing a representation holistically seems to lie in how optimally the void is organized in every representation instance.

The important point here is that such a representation instance is realized through a mixture of icon, index, and symbol. For example, in a musical performance, the symbol is indicated first but then regulated at the level of individual play as an icon and completed as art in terms of index. This happens similarly for visual representations: first is regulation in terms of symbol, realized in an instance and completed in terms of index, in which void speaks eloquently.

\section{Towards a More Human-Oriented Information Representation}

The semiotic conjecture of void in the previous section highlights the following three points:

- The characteristics of void as a sign lie in its holistic nature.

- Void is a typical reciprocal sign, in which the frameworks of Peirce and Saussure become equivalent.

- Void presents signification more at the level of icon and index, which derive from other semiotic classes. This suggests the importance of optimal spacio-temporal placement of each instance of void.

What can these observations bring to HCI and UX?

Above all, with void being reciprocal and holistic, it is a non-trivial target for handling within computational information representation. In [11], I highlighted the difference in the semiotic natures of computer and human signs, in terms of constructive vs. structural. The difference derives from the different interpretive strategies for reflexive expressions in these two sign systems. Natu- 
ral sign systems handle self-reference, including any problematic self-referential expressions, by leaving ambiguity as is. This interpretive mechanism generates a structural system in which the signification of signs exists in the holistic system and the whole sign system operates reflexively. In contrast, in computer sign systems, programs must be constructive, generated by a safe combination of signs that is guaranteed to halt, since self-reference directly concerns the halting problem ${ }^{1}$. Making computer sign systems structural holds the key to developing computer systems that behave in a more human-friendly manner.

Returning to the question raised in Section 1, of how semiotic consideration of void could provoke better information representation, here is a possible answer: by filling the gap between structure and construction for a more humanoriented information representation. Void is a typical sign of the structural type. Naturally, then, void does not conform well with computational handling, and synthesis has been made with the focus on the non-void aspects of information representation. Further, this could be one reason why all such synthesis remains mechanical, in contrast to human representation, which is always adjusted holistically through repeated, reciprocal consideration of non-void and void elements. For example, in automatic musical performance, every length of musical event must be recalculated according to a holistic strategy of how to represent an event and the subsequent void. Likewise, for an interactive system, the optimal spacing of interaction, including speed and silence, requires proper design depending on whom the system interacts with.

Consideration of void in semiotics therefore aids formulation of how signs should be holistically organized in a system - which concerns not only the nonvoid but also the sign system as a whole, including void. This helps fill the gap between structure and construction. How well a representation communicates to an audience depends on the organic handling of signs, including the design of void, which currently tends to be missing and ignored.

There are two possible paths to studying a more human-oriented computational representation:

- holistic analysis of human sign systems, and

- a computational optimization methodology oriented towards holism.

The first point derives from content processing within computer science. It entails construction of various corpora founded on analysis of the content. Such corpus construction, however, has placed the focus on non-void content. It could be possible to integrate void so as to form part of a corpus. This would require assembling a language for describing void, which could be generated through detailed categorical analysis of void and annotation of void by using language appearing in multiple cases of information design ${ }^{2}$. Better synthesis could become possible by using such a corpus as a gold standard.

\footnotetext{
${ }^{1}$ It has been logically proved that any computer based on a Turing machine is incapable of judging whether a given program halts [10].

${ }^{2} \mathrm{~A}$ good starting point could be borrowed from a corpus annotating metalanguage, including elements such as brackets, appearing in [12], in the sense that metalanguage also concerns signification at the semiotic system level.
} 
Second, based on this understanding of void, proper computational handling methods for void must be reconsidered. This is the matter of global optimization of the semiotic system as a whole, including the design of void. Usually an optimization problem requires combinatorial calculation. At the same time, as seen in the previous section, since void has more signification at the level of icon and index, such optimization must be made individually for each instance of representation. Various optimization techniques would apply to synthesis of computational design instances, and moreover, planning algorithms must be deduced from these techniques, in order to construct interactive systems with partially optimized void at each interaction point.

Such studies could highlight the underlying nature of human information. An endeavor towards a better, more human-oriented synthesis could possibly lead to a better man-machine interface.

\section{References}

[1] Casalis, M.: The semiotics of the visible in japanese rock gardens. Semiotica pp. 349-362 (1983)

[2] Cheng, F.: Vide et Plein. Seuil (1991), in French

[3] D'Amato, M.: The semiotics of signlessness: A buddhist doctrine of signs. Semiotica pp. 185-207 (2003)

[4] Köhler, W.: Gestalt Psychology. Liveright Publishing Corporation (1947), reprinted in 1992

[5] Maruyama, K.: Thoughts of de Saussure. Iwanami publishing (1981), in Japanese

[6] Minami, H.: A Study on Ma - A Japanese Aesthetic Representation. Kodansha (1983), in Japanese

[7] Nöth, W.: Handbook of Semiotics. Indiana University Press (1990)

[8] Peirce, C.: Collected Papers. Harvard University Press (1931)

[9] de Saussure, F.: Saussure's Second Course of Lectures on General Linguistics. Pergamon (1908-1909), from the notebooks of Albert Riedlinger and Charles Patois, published in 1998

[10] Sipser, M.: Introduction to the Theory of Computation, 2nd ed. Course Technology (2005)

[11] Tanaka-Ishii, K.: Semiotics of Programming. Cambridge University Press (2010)

[12] Wilson, S.: The creation of a corpus of english metalanguage. pp. 638-646 (2012) 